\title{
Myocardial changes in immature infants requiring prolonged ventilation
}

\author{
D. J. DESA
}

From the Department of Pathology, McMaster University Medical Centre, Hamilton, Ontario, Canada

SUMMARY Fourteen infants who had required prolonged ventilation with high concentrations of oxygen for 14 days or more, were studied at necropsy. 11 infants of immature gestation at birth had gross cardiac hypertrophy, defined as a heart weight greater than 2 SD above the mean for their age. No congenital malformation of valves or septum was found, and in the 7 youngest infants with hypertrophy, the ductus arteriosus was anatomically patent. 3 of these 7 infants had an accompanying murmur. All 11 infants had severe bronchopulmonary dysplasia. The cardiac hypertrophy affected both ventricles and septum in an irregular fashion and was associated with areas of necrosis, progressing to coarse fibroelastotic scars. The intramural vessels showed marked intimal thickening but the main coronary vessels were normal. The 2 youngest infants with cardiac hypertrophy showed the presence of intravascular and endocardial platelet thrombi. In the 3 infants without cardiac hypertrophy less severe zones of necrosis and scarring were present, and only occasional bundles of hypertrophied muscle fibres were seen.

In an attempt to understand these hitherto undescribed lesions, a group of 50 fresh stillbirths and 50 first-week neonatal deaths of comparable gestational age were studied. In 19 of these infants foci of myocardial fibre necrosis were present. It is suggested that the lesions in the older infants represent a more advanced and continuing stage of that seen in the younger infants, and that the foci of necrosis are the result of continuing hypoxia and related problems to a failing coronary circulation. The possibility of myocardial damage represents a serious hazard to the successful therapy of the immature asphyxiated infants.

Congestive cardiac failure and hypertrophy of the right ventricle are relatively common complications in infants who develop bronchopulmonary dysplasia after prolonged ventilation using high $\mathrm{O}_{2}$ concentrations (Rosan, 1975). Though reports of large series of infants with bronchopulmonary dysplasia have been published (Northway, et al., 1967; Bannerjee et al., 1972; Taghizadeh and Reynolds, 1976), myocardial changes were not described in any detail.

This report presents a series of immature, low birthweight infants who were maintained on prolonged ventilation and hyperoxygenation for 14 days or more and who had evidence of cardiac enlargement due to hypertrophy of both left and right ventricles. Several unexpected histological changes in the myocardium were noted, and in an attempt to understand their pathogenesis a group of stillbirths and neonatal deaths occurring within the first week

Received 11 May 1976 of life was collected and histological preparations of the myocardium studied in detail.

\section{Material and methods}

Over a period of 2 years, 14 infants who died after 14 days or more of assisted ventilation and hyperoxygenation were studied at necropsy. All necropsies were conducted personally and the hearts were weighed in a uniform fashion after removal of blood clot from the chambers. Multiple histological blocks were taken from both ventricles and the interventricular septum, processed through to paraffin, and routine sections were stained as appropriate. The remaining pieces of myocardium were stored in $10 \%$ buffered formal saline and small blocks were subsequently removed from the formalfixed material, placed in $3 \%$ glutaraldehyde, postfixed in osmium tetroxide, and embedded in epon. Sections were cut $0 \cdot 5-1 \mu \mathrm{m}$ thick and stained with toluidine blue. Further thin sections were cut with 
an LKB ultramicrotome, stained with lead citrate and uranyl acetate, and examined with a Philips 300 electron microscope.

Details of gestation, birthweight, and other relevant clinical features were taken from the clinical records of the infants who showed cardiac hypertrophy and tabulated. In view of the findings in the older infants a group of 50 fresh stillbirths and $\mathbf{5 0}$ early neonatal deaths, dying within the first week of life, of comparable gestational age to the older infants, were collected from the records of Hamilton, Ontario hospitals (St. Joseph's Hospital and Henderson General Hospital). Sections of the myocardium were studied and where possible fresh blocks of myocardium were sampled. When necessary deeper blocks through the myocardium were cut and stained as appropriate.

Finally, in view of the changes found in the infants with hypertrophy, the myocardial sections from the 3 infants without significant cardiac hypertrophy were reviewed. This included cutting fresh blocks, and multiple sections of both old and new myocardial blocks were studied.

\section{Results}

Table 1 gives the clinical details of the 14 infants studied. The 11 (group 1) infants who showed gross evidence of cardiac hypertrophy are described more fully below.

\section{Clinical details.}

Gestation. All of the 11 infants were under 35 weeks' gestation. 7 of the 11 had a gestational age of 28 weeks or less, while 4 infants had a gestational age of between 29 and 34 weeks.

Birthweight. Only one infant weighed more than $1500 \mathrm{~g}, 6 \mathrm{had}$ a birthweight of $1000 \mathrm{~g}$ or less, and 4 between 1001 and $1500 \mathrm{~g}$. In four instances pregnancy was complicated by antepartum haemorrhage, in three instances with toxaemia and hypertension (one with an additional complicating haemorrhage and one with underlying maternal diabetes). Prolonged rupture of membranes was noted as the only anomaly of pregnancy in 2 infants. One infant was the second of twins and in one case no abnormality of the pregnancy other than onset of labour at an immature gestation was noted.

Apgar scores. 6 infants had Apgar scores of 6 or less at 5 minutes, while 10 had Apgar scores of less than 6 at 1 minute.

Indication for ventilation with oxygen. In 10 infants ventilation and hyperoxygenation were needed to treat the respiratory distress syndrome, while in the remaining infant a large omphalocele was present which required surgical correction. Postoperatively the infant required assisted ventilation due to an underlying pulmonary hypoplasia.

Ventilation procedures. All the infants received intermittent positive pressure ventilation (IPPV) with a median peak airway pressure of $45 \mathrm{~cm} \mathrm{H}_{2} \mathrm{O}$ (range $25-70 \mathrm{~cm} \mathrm{H}_{2} \mathrm{O}$ ), provided through a Bourns LS-150 respirator. Positive expiratory end pressure (PEEP) was provided for all during the use of IPPV, and with some variation PEEP was used for about half the time the infant was on IPPV. The maximum PEEP used varied from $1-11 \mathrm{~cm} \mathrm{H}_{2} \mathrm{O}$ (median 5 $\mathrm{cm} \mathrm{H}_{2} \mathrm{O}$ ). The duration of ventilation with oxygen concentrations greater than $40 \%$ and $80 \%$ are shown in Table 1.

Range of ages. The infants' ages ranged from 24 to 184 days. However, they were divided into three sets according to their age: 4 infants aged 24 to 46 days: 3 infants aged 99-102 days; and 4 infants aged 161 to 184 days.

In all the infants repeated episodes of apnoea and bradycardia were noted and these episodes were accompanied by varying degrees of hypoxia. There was no clinical or serological evidence of any viral infection or myocarditis.

Associated pulmonary and vascular pathology. All of the 11 infants showed severe changes of bronchopulmonary dysplasia (grades III and IV of Rosan, 1975). In addition to the parenchymal damage, gross pulmonary arteriolar medial hypertrophy was noted in all the infants. In 8 of the 11 infants organizing mural thrombi were present within the pulmonary arterial tree.

The ductus arteriosus was patent in 3 infants and an accompanying murmur had been noted clinically. In the remaining 4 younger infants ( $<102$ days of age) the ductus arteriosus was of probe patency at necropsy. In the 4 oldest infants of the group the ductus arteriosus was obliterated. Varying degrees of organizing thrombosis and fibrous elevated plaques related to catheterization were noted in all the infants, and these infants form part of a series of thromboatheromatous complications of umbilical arterial catheterization (Tyson et al., 1976).

Cardiac changes. In all the 11 infants the coronary vessels were normal and no malformation of the valves or septum was noted. Cardiac hypertrophy was defined as representing a weight greater than the 'mean +2 SD' for the age after correction for 
the immature gestation of the infant using the data of Schulz et al. (1962). When the mean +2 SD for the corrected age of the infant is known it is possible to express the heart weight as a percentage increase over the mean $+2 \mathrm{SD}$. When the weights of the hearts of the affected infants were expressed in this way (Fig. 1) a tendency was shown for the degree and severity of cardiac enlargement to be related to the age of the infant. In Fig. 1 the arrows point to the cases who had a persistent ductus arteriosus at necropsy with an accompanying murmur.

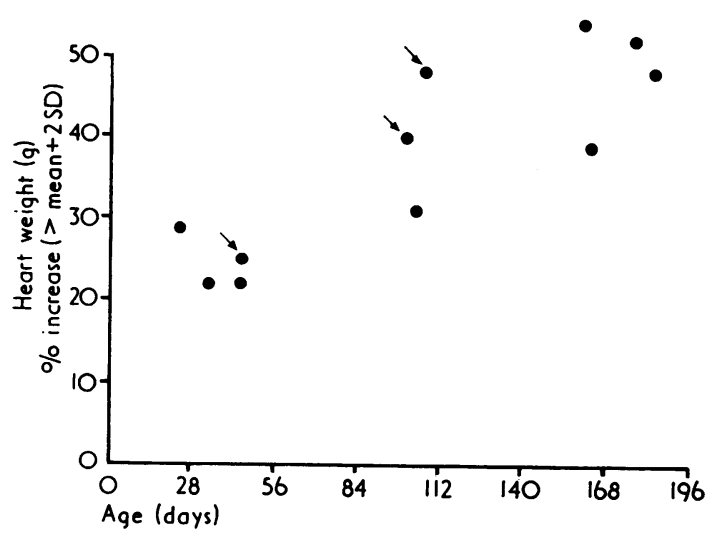

Fig. 1 Heart weights of infants with cardiac hypertrophy plotted, as percent increase over the expected meant $2 S D$ against their postnatal age. Arrows indicate 3 infants with a murmur accompanying the presence of $a$ persistent ductus arteriosus at necropsy. An apparent exaggeration of the cardiac hypertrophy with increasing age can be seen.
Histological changes. The most striking feature was the presence of fascicles of hypertrophied fibres in both ventricles, with swollen bizarre nuclei present below the epicardium, endocardium, and within the main mass of the myocardium. The distribution of hypertrophied fibres was irregular and varied from case to case, but the changes were best seen in the interventricular septum. While there was a tendency for the right ventricle to be more severely affected than the left, the changes were not confined to this ventricle.

Nuclear changes in hypertrophied myocardial fibres. The grossly distorted and enlarged nuclei were readily visible on routine preparations. In $1 \mu \mathrm{m}$ sections stained with toluidine blue, they had an extremely complex configuration with irregular prolongations (Fig. 2). These changes were even more striking in ultrastructural preparations, where the bizarre shapes could be seen to be related to marked infolding of the nuclear membrane (Fig. 3). The state of preservation of the other organelles, however, was unsatisfactory and a detailed assessment was not possible.

Necrosis and scarring. In the younger infants in group I, multiple small foci of myocardial fibre necrosis were identified (Fig. 4). Similar bands of necrotic fibres were seen in the older infants, but more detailed search for these changes was usually necessary. While the necrotic fibres were predominantly in the subendocardial zones, they were not restricted to this area, and patchy areas of necrosis could be seen in deeper layers of the myocardium. In several infants, however, necrosis was particularly

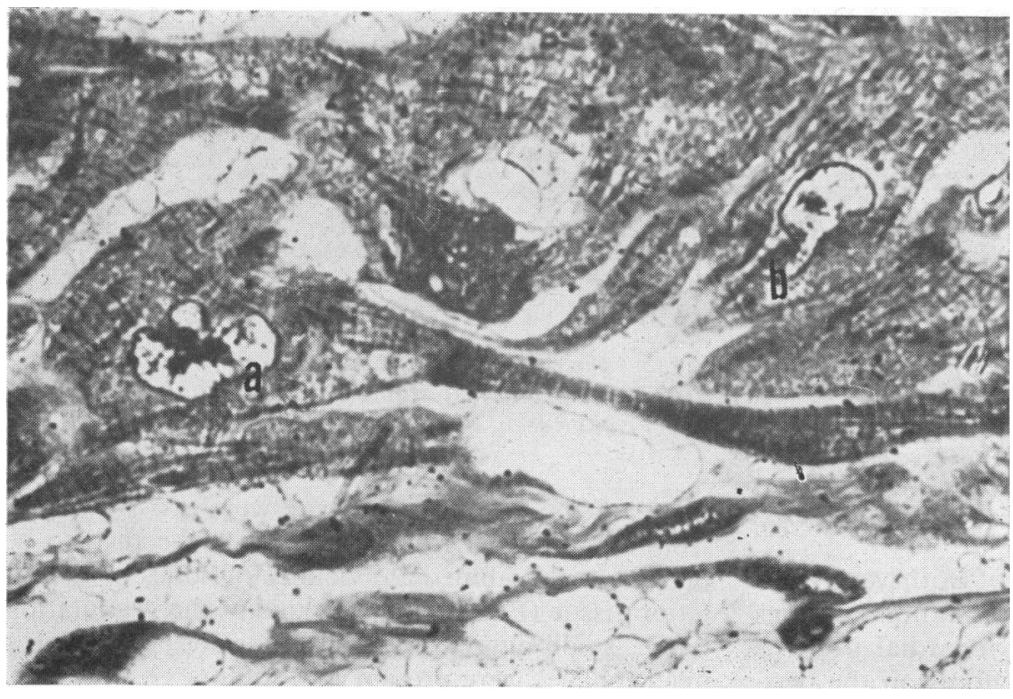

Fig. 2 Case 5. Grossly irregular nuclei resembling cloverleaf forms (a) and with elongated projections (b), characteristically seen in the hypertrophied myocardial fibres. Note the presence of scarring visible in the lower third of the field. ( $1 \mu \mathrm{m}$, epon-embedded, toluidine blue-stained section. $\times 660$.) 


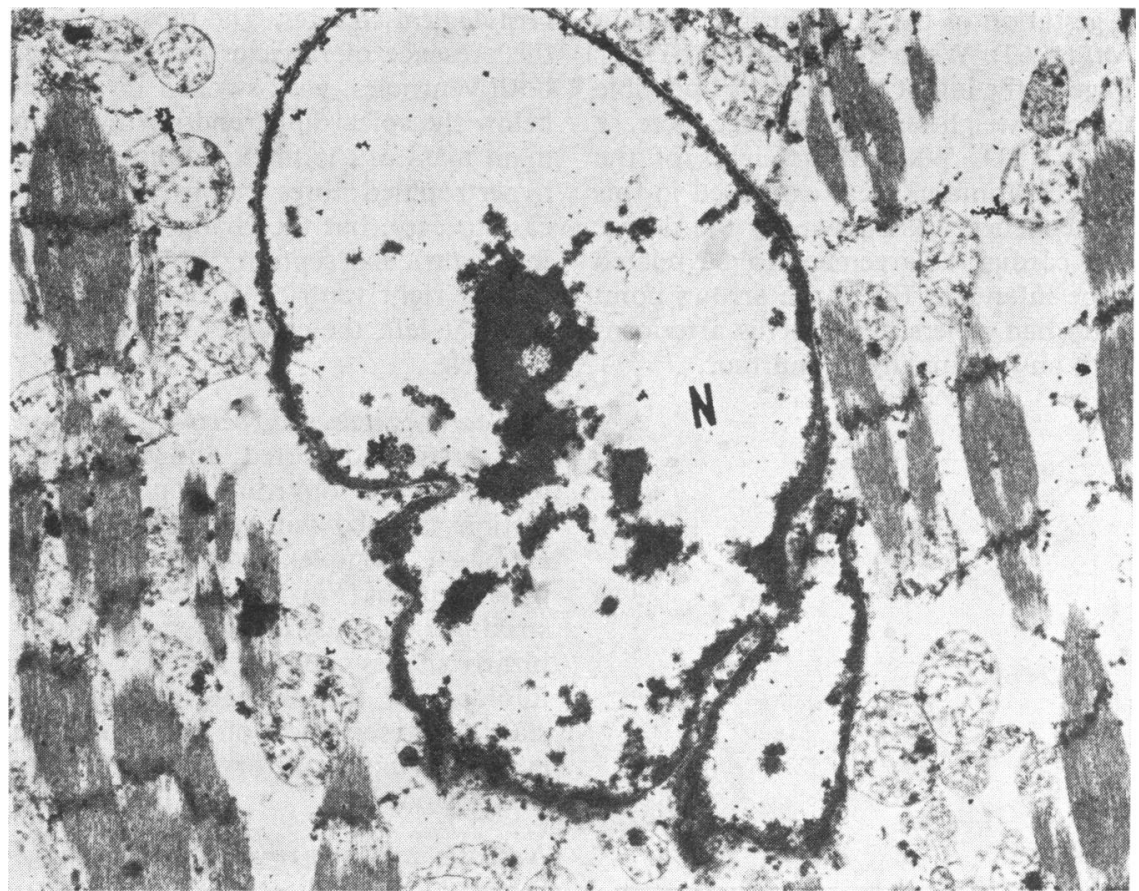

marked in the papillary muscles of both ventricles. Foci of granulation tissue were common findings on the edge of larger areas of myocardial necrosis (Fig. 5). The healthy myocardial fibres on the peri-

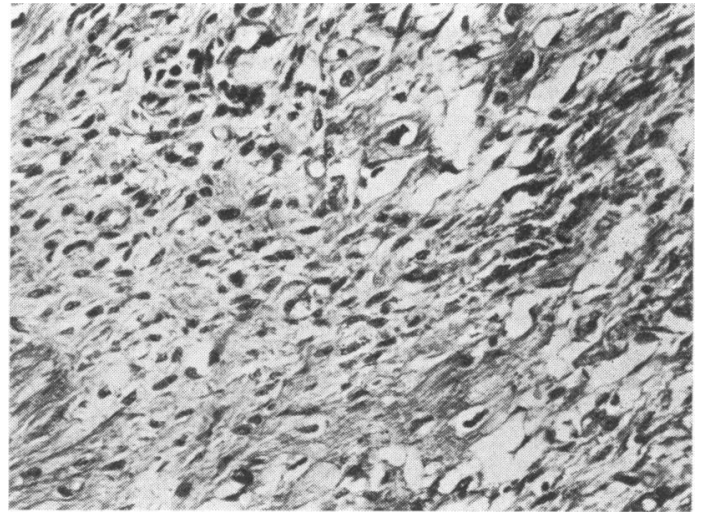

Fig. 5 Case 3. A centrally placed zone of granulation tissue replacing darkly staining necrotic fibres (upper right) can be seen. (Gomori's Trichrome. $\times 140$.)

phery of the granulation tissue were grossly hypertrophied and the most atypical nuclei seemed to be present in the immediate vicinity of zones of granulation tissue (Fig. 6). On the edge of the granulation tissue, small aggregates of haemosiderin-containing macrophages were present in 5 infants. 


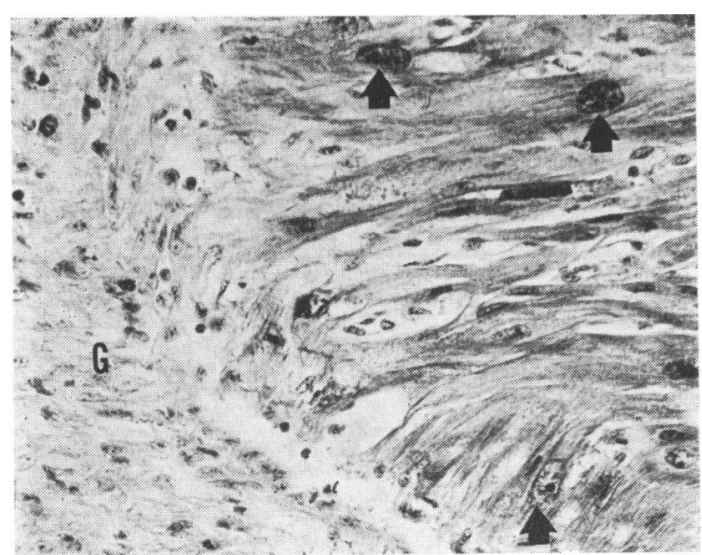

Fig. 6 Case 6. Grossly enlarged nuclei of hypertrophied myocardial fibres (some arrowed) are seen on the edge of a zone of granulation tissue (G). (Gomori's Trichome. $\times 140$.)

Large scars were present in all the infants with cardiac hypertrophy but were most noticeable in the oldest infants (Fig. 7). Finer fibrous scars around individual fibres and small groups of myocardial fibres were identified readily in all the 11 infants, particularly around the periphery of the larger areas of scarring. Structures with the tinctorial properties of elastic fibres were plentiful within the older areas of scarring, and it would be more accurate to describe the scars as being fibroelastotic in character. Within the scars small clusters of thin-walled capillaries could be seen. Collars of surviving myocardium around blood vessels on the edge of larger scars were

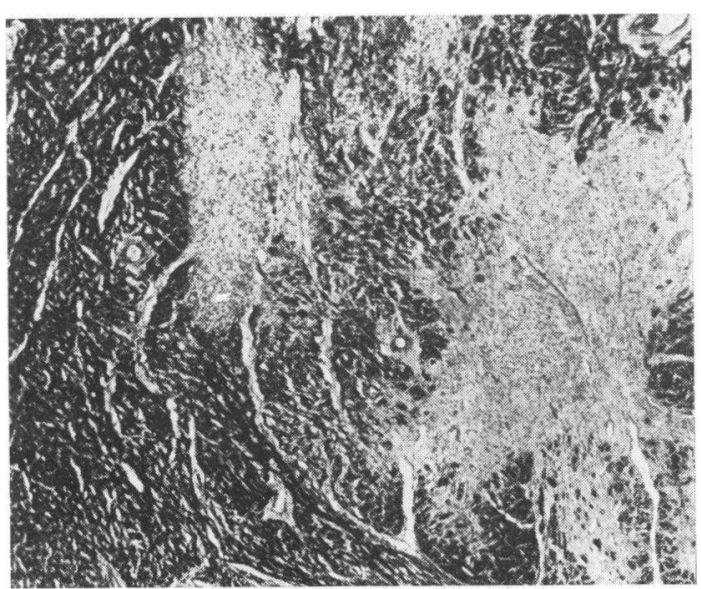

Fig. 7. Case 7. Large fibroelastotic scars present in the grossly thickened septum are clearly seen. A perivascular cuff of surviving myocardial cells is seen in the centre. (Gomori's Trichrome. $\times 25$.) often seen and a partial 'myocardial cuff' is shown in Fig. 7. In 2 of the oldest infants with cardiac hypertrophy, small clusters of cells closely resembling fat cells were seen within the myocardial scars (Fig. 8). In another infant small bundles of mineralized myocardial fibres were identified in the base of a scarred, papillary muscle (Fig. 9). Small plaques of endocardial fibroelastosis were seen in 8 of the 11 infants.

It was apparent that in the 11 infants with cardiac hypertrophy a mixture of myocardial necrosis and fibroelastotic scarring was present. In general, necrosis was noted more readily in the younger

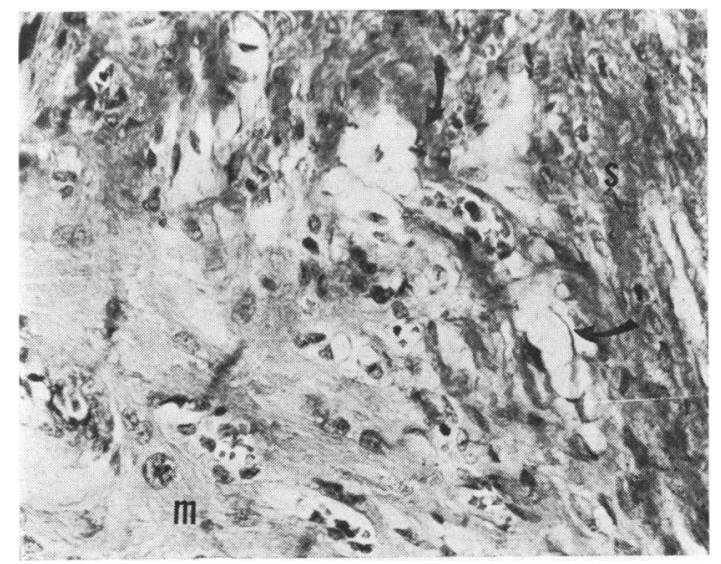

Fig. 8. Case 10. Small clusters of fat cells (arrows) on the edge of a large scar $(S)$. Note the numerous dilated capillaries and the grossly enlarged nuclei within a distorted tangle of myocardial fibres $(m)$. (Van Gieson stain. $\times 150$.)

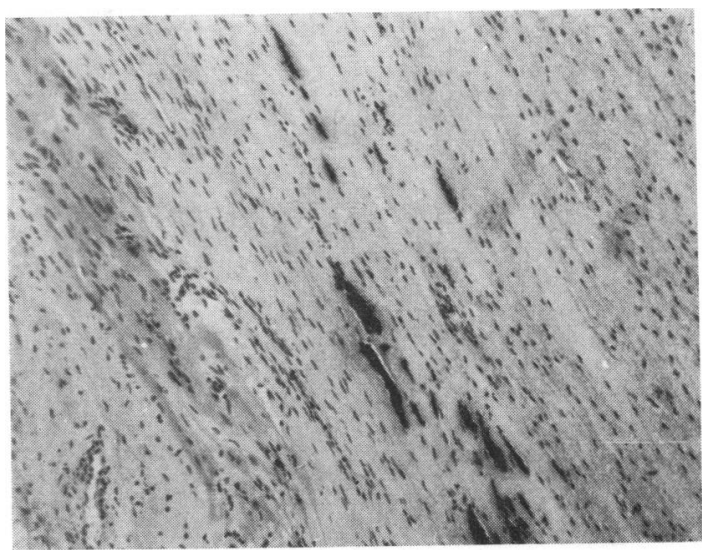

Fig. 9. Case 8. Darkly staining calcified fibres present in the base of a scarred papillary muscle. (Haematoxylin and eosin. $\times 80$.) 
infants, while scarring was the predominant feature in the older. However, an overlap between these two extremes was present in all 11 infants. The variation seen in any one case suggested that necrosis and scarring were a continuing process in all the infants.

Vascular changes. Since without exception the main coronary arteries were histologically normal, the intimal proliferation seen in the intramural myocardial arteries were particularly striking. The intimal changes were most severe in the older infants and the degree of severity appeared to be related to the age of the infant. In the 2 youngest infants of the series small platelet thrombi were present in small intramural vessels (Fig. 10) and in these 2 infants microscopic thrombi were present on the endocardium (Fig. 11) but there were no vegetations on the valves.

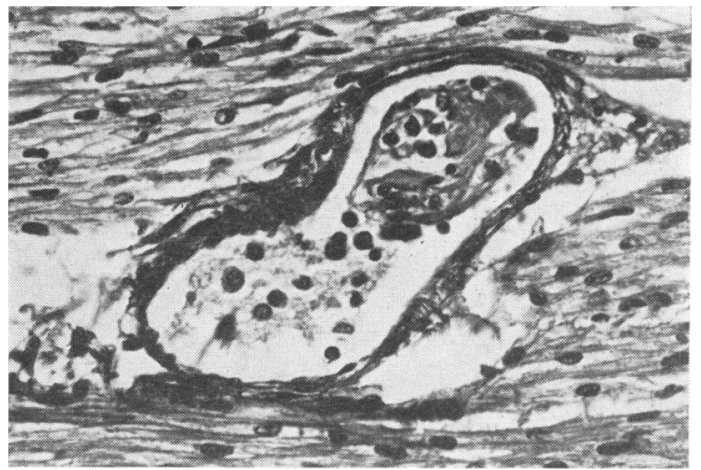

Fig. 10 Case 2. Small platelet thrombi present in a smaller intramural arterial vessel. The myocardial fibres and nuclear size in this field are normal. (Haematoxylin, Alcian Green, Van Gieson. $\times 225$.)

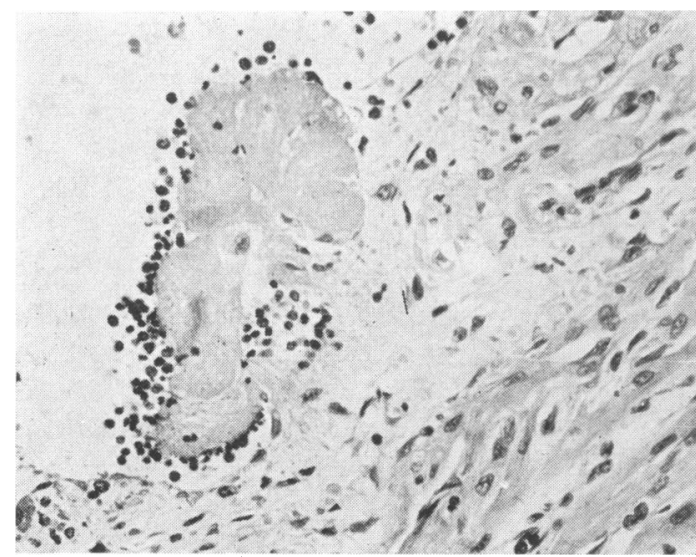

Fig. 11 Case 1. An endocardial thrombotic, platelet vegetation in the left ventricle. (Haematoxylin and eosin. $\times 150$.)
Myocardial changes in stillbirths and early neonatal deaths. A summary of the findings in the myocardium of fresh stillbirths and neonatal deaths of 7 days or less in age is shown in Table 2. In 9 of the

Table 2 Myocardial changes in stillbirths and neonatal deaths $(7 d)$

\begin{tabular}{lcc}
\hline & $\begin{array}{c}\text { Stillbirths } \\
(n=50)\end{array}$ & $\begin{array}{c}\text { Neonatal deaths } \\
(n=50)\end{array}$ \\
\hline No lesion & 41 & 20 \\
Petechial haemorrhage only & 4 & 16 \\
Petechiae + fibre necrosis & $5^{*}$ & $14^{*}$ \\
Antepartum haemorrhage & 4 & 9 \\
Toxaemia hypertension & 1 & 3 \\
$\begin{array}{l}\text { Maternal urinary tract } \\
\text { infection }\end{array}$ & - & 1 \\
Not known & - & 1 \\
\hline
\end{tabular}

"Complications of pregnancy shown in affected infants with myocardial necrosis (lower half).

stillbirths scattered petechial haemorrhages could be identified, while petechiae were present in $\mathbf{3 0}$ of the early neonatal deaths. In 5 of the 9 stillbirths with petechial haemorrhages small foci of myocardial fibre necrosis could be identified, and in 14 of the 30 neonatal deaths similar necrotic foci were seen. In the most severe case (Fig. 12) gross areas of

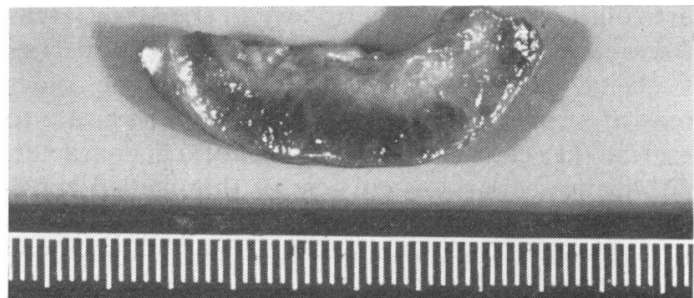

Fig. 12 Male infant, 32 weeks, $1380 \mathrm{~g}$, maternal antepartum haemorrhage, aged 3 days. Massive areas of necrosis are seen in the wall of the left ventricle (upper half). The necrotic areas show up clearly as fuchsinophilic areas (arrowed) in the histological section (lower half). (Modified acid fuchsin stain. Scale in $\mathrm{mm}$.) 
myocardial necrosis and haemorrhage were readily identifiable but in most cases only small isolated bands of necrotic fibres could be seen (Fig. 13). In one infant large geographic areas of necrosis within a papillary muscle were readily recognizable.

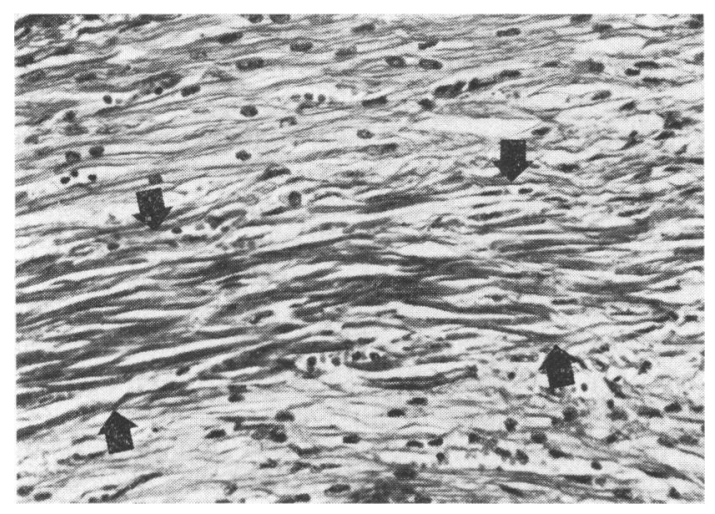

Fig. 13 Male infant, 26 weeks, $880 \mathrm{~g}$, maternal antepartum haemorrhage, aged 1 day. Small bands of necrotic fibres present in the right ventricle (arrowed). (Picromallory. $\times 150$.)

In adddition to the incidence of myocardial fibre necrosis, the major complications of pregnancy are shown in Table 2 . There is considerable similarity between the nature of the complications most frequently noted in the stillbirths and neonatal deaths and those seen most commonly in group I infants which formed the basis of this present report (cf. Tables 1 and 2).

Review of findings in 3 infants in group II (without cardiac hypertrophy). On re-evaluation, it was clear that all 3 infants showed qualitatively similar changes to those seen in the infants with cardiac hypertrophy. However, the zones of necrosis were smaller and finer and there appeared to be far fewer bands of hypertrophied muscle fibres. In none of the 3 infants without cardiac hypertrophy was there necrosis of a papillary muscle, nor were there large fibrous scars. Vascular changes were much less pronounced.

\section{Discussion}

The changes in the myocardium reported in this paper were unexpected in several ways. It is worth re-emphasizing the qualitative similarity between the changes in the infants in both groups I and II. The lack of any previous detailed descriptions of the myocardium in infants with bronchopulmonary dysplasia made a full understanding of the pathogenesis of the lesions reported here extremely difficult and in some respects speculative. It would, however, be surprising if they were not of considerable clinical significance.

The presence of hypertrophy of the right ventricle secondary to pulmonary hypertension associated with bronchopulmonary dysplasia is understandable. The presence, however, of hypertrophy of the left ventricle, is not as explicable and is reminiscent of the development of left ventricular dysfunction, with or without hypertrophy, occasionallly identified in association with chronic lung disease in adults (Kountz et al., 1936; Spain and Handler, 1946; Michelson, 1960; Rao et al., 1968; Baum et al., 1971).

An obvious consideration in the development of the left ventricular hypertrophy seen in some adults with chronic lung disease is the role of hypoxia and hypercapnia (Fishman, 1971). It is reasonable to suppose that similar considerations apply, and with equal force, in infants despite the presence of assisted ventilation and hyperoxygenation, since many of these infants had episodes of apnoea and bradycardia and long periods with poor arterial oxygen saturation and hypercapnia. Also important was the presence of necrotic zones of myocardium in the hearts of all the infants with a history of prolonged ventilation, irrespective of the presence or absence of hypertrophy. In adults (Mitchell and Schwartz, 1963) it has been shown that at any given level of blood pressure, hearts with large foci of ischaemic damage weigh more than hearts without such damage. Presumably the surviving fibres hypertrophy in a compensatory response by Starling's law. In the neonate identical mechanisms may operate, and it is possible to see one aspect of the development of hypertrophy as a response to areas of myocardial damage.

Among the possible aetiological agents of myocardial damage, the volume of coronary blood flow and vascular patency are crucial. The presence of thrombi in the 2 youngest infants with hypertrophy, and the proliferative intimal lesions in the intramural vessels of the older of the 11 infants indicate some interference with either the volume of coronary blood flow or vascular patency, or both. The integrity of the myocardium is vital to the coronary circulation, and theoretically it would be possible to construct a vicious cycle where myocardial damage (irrespective of the aetiology) could compromise coronary flow and lead to further damage.

It is worth considering some of the elements that could lead to myocardial damage in the ventilated infants. From Table 2 it is clear that several of the infants could have suffered damage to the myocardium at the time of delivery, or shortly thereafter. Gelli et al. (1968) showed that anoxia can produce 
severe ultrastructural changes in the myocardium of rabbit fetuses. In particular, the mitochondrial changes are comparable to those described in irreversible, anoxic myocardial injury in rats (Ganote et al., 1975). To some extent both the ultrastructural changes and reduction of contractility associated with anoxia in perfused isolated rabbit hearts can be counterbalanced by increasing the supply of glucose in the perfusing solution (Gelli et al., 1968; Gennser, 1972). Similarly, the action potential duration in anoxic guinea pig ventricular muscle can be restored to near-normal values by increasing concentrations of glucose in the medium (MacLeod and Prasad, 1969). Lactic acidosis can also produce severe changes in myocardial mitochondria in experimental animals (Armiger et al., 1975), and there is some evidence that in comparable conditions of organ culture the hearts of immature fetuses release proportionately more lactate into the culture medium than mature fetuses (Wildenthal, 1973). A very early change described as a 'zonal lesion' by Ratliff et al. (1975), has been considered of vital importance in the genesis of hypovolaemic shock.

In the material available in the present study the poor state of preservation did not permit any identification of subtle and delicate structural changes, and the significance of finding such early structural changes in necropsy material is a moot point. There are grounds for believing, however, that the anoxic, shocked, hypoglycaemic, immature infant is a candidate for poor myocardial function leading to necrosis of the myocardium, particularly since such a stressed state may be associated with increased platelet aggregation (Haft and Fani, 1973).

In addition to the functional factors that could compromise myocardial function, and thereby structure, anatomical features could also play an important role, foremost being the presence of the ductus arteriosus. In cases of persistent ductus arteriosus in older infants, hypertrophy of the left ventricle is well-known, and the presence of a persistent ductus arteriosus is likely to help explain the involvement of both ventricles in the infants in this series. While the ductus arteriosus had been obliterated at the time of necropsy in the oldest infants, it may have been patent for some time postnatally. The direction of shunting through such a persistent ductus arteriosus would be expected to be right-to-left if the pulmonary arterial resistance had been raised by the presence of bronchopulmonary dysplasia with associated pulmonary hypertension. In the presence of focal necrosis of the left ventricle, it is likely that the right-to-left shunt is exaggerated, as in the infant reported by Guller and Bozic (1972). Therefore, in the presence of myocardial damage the deleterious effects of a persistent ductus arteriosus might be considerably accentuated.

Involvement of papillary muscles was a common finding in the hearts of the infants in this series. The significance of the involvement of papillary muscles is uncertain, but it may be associated with poor valve function in affected infants, as it is in affected adults. Clearly, necrosis of a papillary muscle would constitute an additional potential hazard in an already compromised state.

Nuclear changes in hypertrophied cardiac muscle cells have been discussed in considerable detail by Ferrans et al. (1975) and they indicate the synthesis of excessive amounts of nuclear membrane in response to the stimulus of cardiac hypertrophy. The redundant nuclear membrane becomes folded and produces the bizarre configurations noted earlier. In a subsequent report the same group (Maron et al., 1975) describe degenerative changes in the hypertrophied muscle cells, and suggest that some of the lesions seen could conceivably interfere with contractile function. The mere presence therefore of hypertrophied fibres need not signify an adequate compensation for previous damage.

Despite the lack of an adequate ultrastructural study it is clear that various severe myocardial changes are to be expected in some anoxic, hypoglycaemic, immature infants. With the increasing survival rate of such infants it is possible that the myocardial lesions may assume a greater importance. Since an essential feature of resuscitation from asphy$\mathrm{xia}$ is an adequate cardiovascular response (Cross, 1966), it is reasonable to suggest that the lesions seen in this small series of infants could be regarded as a handicap to resuscitation, and a hazard to be considered seriously if intensive therapy is to be successful.

I am grateful to my colleagues in the Departments of Laboratory Medicine in St. Joseph's and Henderson General Hospitals, Hamilton, for their co-operation and for access to their case material, and to Dr. O. K. Bhakoo, now in the Department of Paediatrics, Postgraduate Medical Institute, Chandigarh, India, for access to data on the ventilation therapy in the older infants.

\section{References}

Armiger, L. C., Seelye, R. N., Elswijk, J. G., Carnell, V. M., Benson, D. C., Gavin, J. B., and Herdson, P. B. (1975). Mitochondrial changes in dog myocardium induced by lactate in vivo. Laboratory Investigation, 33, 502-508.

Bannerjee, C. K., Girling, D., and Wigglesworth, J. (1972). Pulmonary fibroplasia in newborn babies treated with oxygen and artificial ventilation. Archives of Disease in Childhood, 47, 509-518. 
Baum, G. L., Schwartz, A., Llamas, R., and Castillo, C. (1971). Left ventricular function in chronic obstructive lung disease. New England Journal of Medicine, 285, 361365.

Cross, K. W. (1966). Resuscitation of the asphyxiated infant. British Medical Bulletin, 22, 73-78.

Ferrans, V. J., Jones, M., Maron, B. J., and Roberts, W. C. (1975). The nuclear membranes in hypertrophied human cardiac muscle cells. American Journal of Pathology, 78, 427-446.

Fishman, A. P. (1971). The left ventricle in "chronic bronchitis and emphysema'. New England Journal of Medicine, 285, 402-404.

Ganote, C. E., Seabra-Gomes, R., Nayler, W. G., and Jennings, R. B. (1975). Irreversible myocardial injury in anoxic perfused rat hearts. American Journal of Pathology, 80, 419-438.

Gelli, M. G., Ericsson, J. L. E., and Enhörning, G. (1968). ECG compared with myocardial ultrastructure in anoxic foetuses of normal and hyperglycaemic rabbits. Acta Paediatrica Scandinavica, 57, 330-338.

Gennser, G. (1972). Influence of hypoxia and glucose on contractility of papillary muscles from adult and neonatal rabbits. Biology of the Neonate, 21, 90-106.

Guller, B., and Bozic, C. (1972). Right-to-left shunting through a patent ductus arteriosus in a newborn with myocardial infarction. Cardiology, 57, 348-357.

Haft, J. I., and Fani, K. (1973). Intravascular platelet aggregations in the heart induced by stress. Circulation, 47, 353-358.

Kountz, W. B., Alexander, H. L., and Prinzmetal, M. (1936). The heart in emphysema. American Heart Journal, 11, 163-172.

MacLeod, D. P., and Prasad, K. (1969). Influence of glucose on the transmembrane action potential of papillary mu icle. Effects of concentration, phlorizin and insulin, non-metabolizable sugars and stimulators of glycolysis. Jcurnal of General Physiology, 53, 792-815.

Maron, B. J., Ferrans, V. J., and Roberts, W. C. (1975). Ultrastructural features of degenerated cardiac muscle cells in patients with cardiac hypertrophy. American Journal of Pathology, 79, 387-413.

Michelson, N. (1960). Bilateral ventricular hypertrophy due to chronic pulmonary disease. Diseases of the Chest, 38, $435-446$.
Mitchell, J. R. A., and Schwartz, C. J. (1963). The relation between myocardial lesions and coronary artery disease. II. A selected group of patients with massive cardiac necrosis or scarring. British Heart Journal, 25, 1-24.

Northway, W. H., Jr., Rosan, R. C., and Porter, D. Y. (1967). A new syndrome of pulmonary disease following respiratory therapy of hyaline membrane disease: bronchopulmonary dysplasia. New England Journal of Medicine, 276, 357-368.

Rao, B. S., Cohn, K. E., Eldridge, F. L., and Hancock, E. W. (1968). Left ventricular failure secondary to chronic pulmonary disease. American Journal of Medicine, 45, 229-241.

Ratliff, N. B., Kopelman, R. I., Goldner, R. D., Gruz, P. T., and Hackel, D. B. (1975). Formation of myocardial zonal lesions. American Journal of Pathology, 79, 321-328.

Rosan, R. C. (1975). Hyaline membrane disease and a related spectrum of neonatal pneumopathies. Perspectives in Pediatric Pathology, Vol. 2, p. 15. Ed. by H. S. Rosenberg and R. P. Bolande. Year Book Medical Publishers, Chicago.

Schulz, D. M., Giordano, D. A., and Schulz, D. H. (1962). Weights of organs of fetuses and infants. Archives of Pathology, 74, 244-250.

Spain, D. M., and Handler, B. J. (1946). Chronic cor pulmonale: sixty cases studied at necropsy. Archives of Internal Medicine, 77, 37-65.

Taghizadeh, A., and Reynolds, E. O. R. (1976). Pathogenesis of bronchopulmonary dysplasia following hyaline membrane disease. American Journal of Pathology, 82, 241-258.

Tyson, J. E., deSa, D. J., and Moore, S. (1976). Thromboatheromatous complications of umbilical arterial catheterization in the newborn period: a clinicopathological study. Archives of Disease in Childhood, 51, 744-754.

Wildenthal, K. (1973). Studies of foetal mouse hearts in organ culture: metabolic requirements for prolonged function in vitro and the influence of cardiac maturation on substrate utilization. Journal of Molecular and Cellular Cardiology, 5, 87-99.

Correspondence to Dr. D. J. deSa, Department of Pathology, McMaster University Medical Centre, 1200 Main Street West, Hamilton, Ontario L8S 4J9, Canada. 